
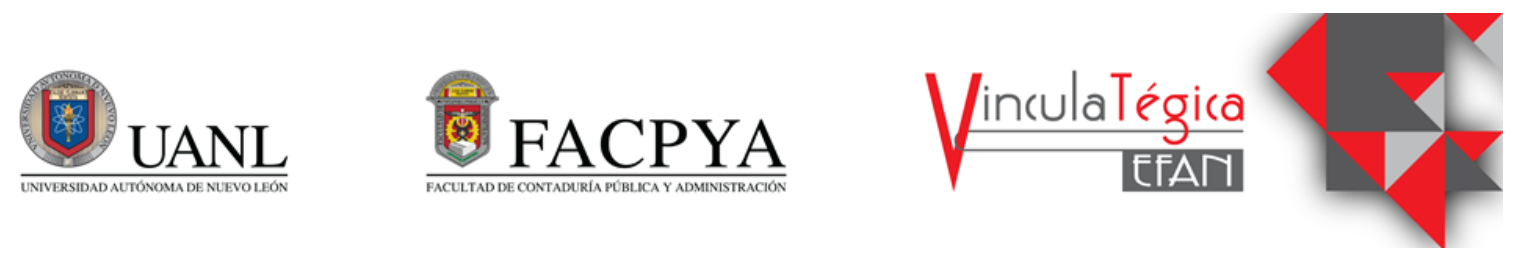

\title{
Bienestar laboral en docentes de una institución pública
}

\author{
Jonathan Nahum de Jesús Álvarez ${ }^{1}$ y Margarita del Consuelo Santiago Bautista ${ }^{2}$ \\ ${ }^{1}$ Universidad Veracruzana campus Coatzacoalcos (México) jdejesus@uv.mx, Dirección: Av. Universidad \\ Km. 7.5 Coatzacoalcos, Veracruz, México. CP. 96538, Ciudad; Coatzacoalcos, México, Teléfono: \\ (922) 1346897. \\ ${ }^{2}$ Universidad Veracruzana campus Coatzacoalcos (México) mcsb.1997@outlook.es,Dirección: Av. \\ Universidad Km. 7.5 Coatzacoalcos, Veracruz. México. CP. 96538, Ciudad; Coatzacoalcos, México, \\ Teléfono: (922)116 0231.
}

Información del artículo revisado por pares

Fecha de aceptación: junio-2021

Fecha de publicación en línea: diciembre-2021

DOI: https://doi.org/10.29105/vtga7.1-157

\section{Resumen}

Este trabajo presenta los resultados de una investigación dirigida en analizar los factores de bienestar laboral en los docentes la Facultad de Contaduría y Administración de la Universidad Veracruzana (UV), todo esto, desde su propia percepción. Esta investigación tiene un carácter analítico, además de tener un corte transversal. Para ello, se utilizó la técnica de encuesta, a través de un cuestionario al que se le denominó: Cuestionario de Bienestar laboral, el cual está estructurado por cinco factores; la primera identifica el logro que el docente proporciona a sus metas y resultados; la segunda, evalúa el factor de reconocimiento que los docentes perciben por parte de su jefe inmediato, el tercer factor mide la perspectiva de su trabajo, es decir, si lo encuentra desafiante, innovador, creativo, entre otros; el cuarto factor es el de identificar su propia responsabilidad en el trabajo y el último factor de promoción relacionado al cambio horizontal o vertical dentro de la organización. La hipótesis que se planteó fue la siguiente: Los factores del bienestar laboral presentes en los docentes de la facultad de contaduría y administración de la universidad veracruzana campus Coatzacoalcos es favorable, se encuestó a 59 docentes de FCA, en la información se observa que los factores son importantes en la percepción del bienestar laboral en el ejercicio de sus propias funciones. Por otra parte, las situaciones contractuales no representan variaciones significativas en su bienestar. Para finalizar, la característica género sí
\end{abstract}

\begin{abstract}
This paper presents the results of an investigation aimed at analyzing the factors of labor well-being in teachers at the Faculty of Accounting and Administration of the Universidad Veracruzana (UV), all this, from their own perception. This research is analytical in nature, in addition to being cross-sectional. For this, the survey technique was used, through a questionnaire called: Labor Wellbeing Questionnaire, which is structured by five factors; the first identifies the achievement that the teacher provides for their goals and results; the second, assesses the recognition factor that teachers perceive from their immediate boss, the third factor measures the perspective of their work, that is, if they find it challenging, innovative, creative, among others; The fourth factor is to identify your own responsibility at work and the last promotion factor related to horizontal or vertical change within the organization. The hypothesis that was raised was the following: The factors of labor well-being present in the teachers of the Faculty of Accounting and Administration of the Veracruz University Coatzacoalcos campus is favorable, 59 teachers of FCA were surveyed, in the information it is observed that the factors They are important in the perception of labor well-being in the exercise of their own functions. On the other hand, contractual situations do not represent significant variations in their well-being. Finally, the gender characteristic did present a significant
\end{abstract}


presentó diferencia significativa para el bienestar laboral. difference for labor well-being.

Palabras clave: Factores de bienestar, Laboral, percepción.

\section{INTRODUCCIÓN}

El bienestar laboral es uno de los temas más trascendentes, importantes y novedosos en los últimos tiempos. El presente trabajo titulado bienestar laboral en docentes de una institución pública, tiene el objetivo de determinar la presencia favorable de los factores de bienestar laboral presente en docentes de la facultad de contaduría y administración, donde se identificaron de manera específica cinco factores relevantes en el área laboral, como son el factor logro, reconocimiento, trabajo en sí, responsabilidad y promoción con los cuales se pretende saber cuál es la presencia de dichos factores en los docentes del género masculino y femenino, así como en los docentes por tipo de contrato, de la facultad de contaduría y administración en el periodo febrero-julio 2020.

El bienestar laboral se define como el "conjunto de juicios valorativos respecto a las reacciones emocionales en relación con el grado en que la propia experiencia en el trabajo es vivida en términos de satisfacción o insatisfacción". M, S, C y Génesis (2010). El bienestar es importante e identificar la presencia en los docentes dará la seguridad de que el trabajo se realiza con esmero y dedicación, sin duda se busca que el docente pueda dar lo mejor en las aulas y así lograr que existan mejores estudiantes y profesionales.

Actualmente, aunque el bienestar laboral es un tema importante en las instituciones y organizaciones, muy pocas veces se da la tarea de analizar la presencia de los diferentes factores que aportan un favorable o desfavorable crecimiento de bienestar de manera especial en las instituciones educativas del nivel superior.

El bienestar laboral tiene una historia importante en el desarrollo de la vida del ser
Keywords: Well-being factors, Labor, perception. JEL: I21, I31, I38, I39

humano, siempre ha existido y siempre existirá es por ello que un es necesario realizar un estudio a detalle, así como, de los aspectos más importantes del pasado y del presente lo cual nos ayudará a comprender el futuro de este elemento muy importante.

Derivado de esta situación se presentan las generalidades del bienestar laboral en docentes, en donde se estudia desde el termino bienestar de manera general hasta lo que significa el bienestar en el enfoque laboral, así como la importancia de la presencia favorable de bienestar, de igual manera se incluye los diferentes tipos de bienestar que existen entre los más destacado como lo es el bienestar físico, mental, social, intelectual y laboral.

Parte importante de la investigación fue el estudio de los factores de bienestar laboral, así como la definición e importancia de ser docente y su perfil profesional en función a su responsabilidad desde que existe el mundo hasta el tiempo actual, con el fin de indagar y resaltar la importancia que tiene el ser docente y también conocer que factores son los que más afectan y repercuten en el desarrollo de sus actividades.

Por otra parte, se trató la normatividad que existe las cuales regulan el funcionamiento correcto de las situaciones laborales en nuestro país, ya que actualmente existen leyes y normas que regulan el bienestar laboral para darle mejor fundamento y respaldar este elemento importante.

Posteriormente, se estudia el bienestar laboral desde los antecedentes mundiales hasta lo más reciente es decir se presentaron casos realizados desde internacionales hasta regionales para conocer la contextualización sobre el tema que abordaremos.

Posteriormente se presentan los datos relevantes donde se identifican la presencia 
del bienestar laboral en algunas instituciones públicas y privadas del nivel superior. Así como instituciones que se dedican a regular $\mathrm{y}$ fomentar el bienestar del docente en las diferentes instituciones.

En la investigación recabada se presentaron datos sobre las instituciones de educación superior existentes y la población joven, así como la población de docentes que existe en determinado periodo, con el fin de entender y conocer la importancia de estudiar el bienestar en docentes del nivel superior.

En la investigación de nivel nacional se encontró dentro de los estudios un documento de investigación adecuado para medir el bienestar laboral en los docentes lo cual fue de apoyo importante para la elaboración del presente trabajo.

También se presentan datos sobre indicadores que regulan el desempeño de los docentes en la universidad veracruzana y de forma específica sobre los resultados obtenidos en la facultad de contaduría y administración para luego realizar un análisis más profundo.

En este sentido, se presenta el objeto y desarrollo del estudio que se utiliza como análisis de investigación. Primeramente, se establece el planteamiento del problema, el cual identifica la problemática a tratar, donde analizamos el objetivo general y los específicos que pretende identificar los factores de bienestar laboral presentes en los docentes de la FCA, UV campus Coatzacoalcos en el periodo febrero-julio 2020, posteriormente se presenta la pregunta de investigación, con la que se espera saber cuál es la presencia de los factores de bienestar laboral en los docentes, la investigación se limitó a investigar la presencia de los factores del bienestar laboral, y se establece una hipótesis que indica que sí existe una presencia favorable.

Para llevar a cabo esta investigación se utilizó una muestra de docentes activos en el periodo mencionado, así como un cuestionario adaptado sobre el bienestar laboral realizado por la facultad de humanidades y aprobado por expertos en la materia, así mismo se realizó la validación de la fiabilidad de los datos obtenidos a través del software IBM SPSS Statistics 26, para posterior a ellos, su análisis y representación en gráficas por objetivos específicos, donde se expresan los resultados, interpretaciones y análisis crítico obtenidos con base al instrumento de recopilación.

\section{MARCO TEÓRICO}

Se presenta el panorama del bienestar laboral, iniciando con el significado de esta palabra desde el aspecto general hasta el laboral, lo cual permite conocer su importancia, la clasificación de los diversos tipos que existen yendo de menor a mayor y los factores que inciden a esta definición ya sea de manera positiva o negativa.

Así como también su evolución, a tal grado de hoy en día cuenta con leyes, normar $y$ artículos que respaldan el lineamiento a seguir, para que cada docente o empleado pueda gozar de un alto grado de bienestar dentro de sus áreas de trabajo.

\subsection{Antecedentes del Bienestar}

La palabra bienestar ha existido desde la creación de este mundo y está directamente relacionado con el ser humano, ya que es el único ser capaz de desarrollarse por medio de emociones, sentimientos y habilidades con las personas que lo rodean. A lo largo del tiempo la palabra bienestar ha cobrado importancia en las relaciones interpersonales.

Como lo define el Diccionario real de la lengua española (2006): "1 m. conjunto de las cosas para vivir bien, $2 \mathrm{~m}$, vida holgada o abastecida de cuánto conduce a pasarla bien y con tranquilidad, 3.m. estado de la persona en el que se hace sensible el buen funcionamiento de actividades".

De acuerdo con lo anterior, el bienestar es el estado del ser humano de sentirse, vivir, estar y realizar sus actividades de una manera tranquila, agradable y optima, además, que es indispensable en las relaciones diarias y a su 
vez que aporta gran apoyo al desarrollo del ser humano en sus diferentes funciones, desde las mínimas hasta las máximas. La palabra bienestar de manera general es un término difícil de alcanzar y con diversas variables de estudio, pero sin duda una de las grandes preocupaciones del ser humano y sobre todo de cada institución u organización.

En definición la palabra bienestar está compuesta por el adjetivo "bien" y el verbo "estar" partiendo de ahí podemos decir que es la condición buena, tranquila, cómoda y grata del ser humano en el cual vive un tiempo real en esta condición, por ello el empleo de ambas palabras forman una definición clara y precisa para definir el bienestar que debe tener de forma individual el ser humano.

A continuación, se estudia la definición sobre el bienestar desde el punto de vista de diversos autores y su aplicación al contexto laboral, ya que actualmente en cualquier institución u organización es necesario alcanzar el máximo nivel de esta palabra, y es una de las demandas actuales del mundo laboral en sus colaboradores, en las diferentes áreas en la que cada uno se desarrolla, ya sea desde el más alto hasta el más bajo rango.

Como lo menciona Brief (1998) "se trata de un estado interno del sujeto que se expresa a través de una evaluación emocional y/o cognitiva de su experiencia en el trabajo con cierto grado o desagrado. También se puede decir que el bienestar laboral tienes dos niveles sea de agrado que se puede entender como el nivel bueno que se disfruta en todo aspecto así mismo se encuentra el nivel desagrado que es la situación de insatisfacción el cual también es expresado en diversas formas".

Por su parte, M, S, C y Genesis (2010) definen al bienestar laboral como el "conjunto de juicios valorativos respecto a las reacciones emocionales en relación con el grado en que la propia experiencia en el trabajo es vivida en términos de satisfacción o insatisfacción".
De acuerdo con B, Bauer y Mansfield (2012), "el bienestar laboral está relacionada con la vida y es considerada como la evolución cognitiva de la satisfacción global que la persona desarrolla en su vida general. Es decir, el bienestar está ligado con aquellas virtudes que el individuo desarrolla en los diversos aspectos, ámbitos de su vida y que lo aplicará posteriormente en su vida laboral".

En opinión de Castro (2000), "quien establece una relación del bienestar general con la personalidad, puesto que, según las investigaciones realizadas por este autor, los estilos de personalidad modulan el patrón de los objetivos vitales de los sujetos, y traen consigo consecuencias que determinan el grado de bienestar psicológico. De acuerdo con esta definición podemos decir que este grado de bienestar primero debe ser psicológico es decir estar presente en la mente de la persona para posteriormente expresarlo en actitudes positivas o negativas".

Los presentes autores consideran que el bienestar laboral está directamente relacionado con la vida del ser humano, es decir con sus valores, emociones y satisfacciones que tiene con lo que realiza diariamente y que se expresa a través de actitudes ya sean positivas o negativas.

Por consiguiente, podemos decir que el bienestar laboral tiene origen en la base importante que tiene el ser humano para alcanzar el máximo nivel de sus necesidades como lo menciona en la pirámide de jerarquías de Maslow (1991), es decir en sus niveles de realización del mismo. 
Figura 1. Jerarquía de las necesidades.

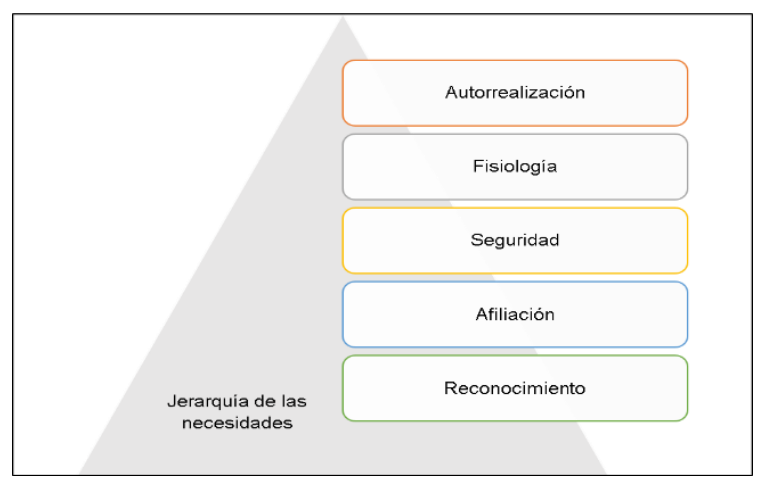

Fuente: Elaboración propia,2020

El primer nivel trata de las necesidades fisiológicas de la persona tales como beber, comer, dormir, etc. Son los instintos más básicos y prácticamente todo el mundo, en la sociedad occidental, tiene cubierto este nivel. Solo hay que ir a la nevera o echarse una siesta para que las necesidades primarias estén cubiertas.

El segundo nivel representa las sensaciones de seguridad. Responde a una necesidad de orden en el mundo, una oportunidad para trabajar, de tener recursos mínimos, de encajar en la sociedad. Una persona que se ha quedado en el paro o que no llega a fin de mes oscilara muy a menudo en este nivel hasta que vuelva a sentir la seguridad de unos ingresos estables.

El tercer nivel es el de las necesidades sociales que se traducen en las ganas de estar en contacto con otra gente. También el amor, la amistad y la familia pertenecen a este grado. Si tenemos amigos que nos cuidan, una familia que nos arropa, un entorno laboral respetuoso y agradable, tenemos nuestras necesidades de afiliación cubiertas y probablemente las damos por sentadas de forma inconsciente.

En el cuarto nivel se encuentra la necesidad de ser apreciado, respetado y de ser alguien importante. Aquí entramos ya en niveles de desarrollo personal más avanzado, en el cual buscamos algo más que el simple hecho de tener nuestras necesidades materiales cubiertas. Todos aquellos que tienen bien asentados los tres primeros niveles pondrán toda su energía y esfuerzo para desarrollar y cubrir este tipo de necesidades de reconocimiento social.

En este sentido, en el nivel más alto se encuentran las necesidades de autorrealización y el desarrollo de las necesidades internas, el desarrollo espiritual, moral, la búsqueda de una misión en la vida, el trabajo y la ayuda desinteresada hacia los demás, etc. De acuerdo con la pirámide de jerarquías dónde nos muestra las necesidades del ser humano en niveles de menor a mayor, podemos decir que el bienestar desde aspectos básico como son los biológicos hasta los de autorrealización que se mencionan son importante $y$ se destacan dentro del desarrollo que cada persona realiza en sus más simples necesidades.

Por lo tanto, de acuerdo a lo que menciona el autor Abraham Maslow, el bienestar laboral abarca dos niveles de esta jerarquía. Se refiere al nivel dos que hace referencia a la necesidad de seguridad en aspectos tan importantes como es el área laboral, sentir o encajar en un ambiente de trabajo y del mismo obtener ingresos, una remuneración por actividades que se realizan. Posteriormente, uno de los niveles que el ser humano que le es importante es la de autorrealización, ya que toda persona desea y anhela sentirse con un propósito en este mundo y más aún alcanzar este propósito como lo es el aspecto laboral.

El término de bienestar laboral es un tema que se ha tratado a través del tiempo como un factor importante en el desarrollo de las instituciones educativas y de cualquier otro giro, es una definición que expande a diversos temas esenciales del bienestar laboral, por lo tanto, su estudio es necesario en el entorno laboral cuándo nos relacionamos con personas que por naturaleza somos distinto y así poder alcanzar el máximo nivel y lograr los objetivos que la misma tenga.

\subsection{Importancia del Bienestar Laboral}

De acuerdo con Zapata, Peralta y Fernández 
(2014) "El estudio del bienestar de los trabajadores se refiere principalmente a las condiciones del entorno laboral como medida de éxito; la cual permite alcanzar los objetivos definidos por las organizaciones".

Como lo menciona Infocop (2017) "Las empresas son el lugar físico en el que las personas pasan la mayor cantidad de tiempo; por lo tanto, las condiciones y características particulares de estos ambientes repercuten en la salud física y mental de los trabajadores, influyendo no solo en el bienestar físico y mental que experimentan, sino en múltiples aspectos de la vida personal y la vida laboral'.

Estos autores coinciden que el bienestar laboral en su nivel de importancia es afectado por aspectos dentro de la misma organización como lo es el ambiente laboral y aspectos externos que afectan de manera directa su estabilidad física y emocional, para que el trabajador se sienta capaz de alcanzar el estándar de metas y objetivos de la institución, y de ahí se desglosa el que él pueda sentirse a gusto en el trabajo o en su defecto tener insatisfacción laboral.

Por su parte Calderón, Murillo y Torres (2003) indican que: "El bienestar docente, porque a pesar de todos los inconvenientes reflejados podemos afirmar que la profesión docente es la más reconfortante de las otras profesiones. Existe un dicho que menciona que detrás de un gran hombre, siempre hay una gran mujer, también podemos aplicar este dicho a la relación que hay entre un alumno y un docente, es decir, que detrás de un gran hombre o de una gran mujer, siempre hay un maestro o una maestra, un docente que impulso el trayecto de su formación académica y otros aspectos relevantes de su vida. La motivación que requiere la construcción de un gran profesional, de una gran persona precisa de un aliento infantil y juvenil muy fuerte y eso, sólo un profesional de la docencia, será capaz de impulsar, evidencia de todo esto es que los docentes frecuentemente somos abordados por alumnos, por alumnas que pasados los años recuerdan la imborrable huella positiva que, en ellos o ellas dejamos".

De lo anterior, se puede analizar la importancia que tiene el bienestar laboral en el docente, y lo necesario que es alcanzar un bienestar laboral en su máximo nivel, ya que es él quien se relaciona diariamente con los alumnos, jóvenes y señoritas que en más de alguna ocasión se acercan a ellos para recibir o para tener también bienestar en diversos aspectos de su vida, y es ahí cuando el docente debe estar preparado no solo para impartir un tema teórico sino también para responder a las necesidades de sus alumnos.

Al criterio de Rodgers, Lee, Swepston, y Van (2009), "Es la necesidad urgente de mejorar las terribles condiciones de trabajo que sufrían muchos trabajadores en los primeros decenios de la revolución industrial. Las largas jornadas laborales en entornos insalubres, el uso no controlado de materiales y equipos peligrosos, el empleo generalizado de niños y unos salarios que mantenían a los trabajadores y a sus familias en la miseria escandalizaron a los pensadores progresistas y movilizaron la acción política a lo largo del siglo XIX”.

Según lo indica Pujol (2016) desde mediados del siglo XX los esfuerzos notables de la academia se han dirigido a querer comprender los motivos por los cuales los empleados experimentan satisfacción en su trabajo, así como sus efectos sobre distintos indicadores de la efectividad y el éxito organizacional. En este sentido, son varios los estudios empíricos que han confirmado la existencia de bucles virtuosos entre este fenómeno y otras variables, se tratan de actitudes (involucramiento con el trabajo, compromiso organizacional, lealtad organizacional) o de resultados (desempeño, ausentismo, rotación, accidentes de trabajo). Sin embargo, y con el transcurrir de los años, la satisfacción laboral dejó de ser importante sólo por su probado impacto sobre la performance, y ya desde una perspectiva más centrada en la salud ocupacional, comenzó a ser abordada como un fenómeno en sí mismo 
valioso por su incidencia sobre el bienestar y la calidad de vida de los empleados.

\subsection{Factores del Bienestar Laboral}

El bienestar laboral se ve afectado por diversos factores que influyen en los empleados al momento de desarrollar sus funciones, dentro de lo que destacan según Hernández y Moreno (2013) "El factor logro, factor reconocimiento, factor trabajo en sí, factor responsabilidad y factor de promoción".

Según lo menciona Preciado (2006) "El factor de logro se define como la capacidad productiva de un individuo que se define y mide en términos de desempeño en un determinado texto laboral y no solo de conocimientos, habilidades, destrezas y actitudes; éstas son necesarias, pero no suficientes por sí misma, para un desempeño efectivo".

El segundo factor de bienestar, lo define Parker, McAdams y Zielinski (2013), al factor de reconocimiento como: "El desempeño excelente tanto ordinarios como extraordinarios, que muestran el compromiso diario de personas y equipos con la excelencia, rechazando lo estándares por debajo de lo normal, y que se reconozca esta buena voluntad para ayudar".

El tercer factor ( trabajo en sí), la revista Redacción de recursos humano (2019) hace énfasis a todas "las sensaciones que tiene cada empleado en su entorno laboral, las cuales pueden ser positivas, negativas o neutrales, y puede ser un factor que influye directamente en los resultados de la organización. Entender lo que los colaboradores sienten respecto a la empresa, su equipo de trabajo y el entorno dentro de la organización permite a las áreas de recursos humanos implementar acciones para mejorar el ambiente laboral, la motivación y la productividad".

El cuarto factor (responsabilidad), Por parte del autor Serrat (2017) indica que " $L a$ responsabilidad es el proceso decisorio y en los resultados, darle el poder, la autoridad, la autonomía, la facultad y la autoridad de tomar decisiones por cuenta propia, con la confianza que esta delegación logrará igualmente alcanzar los resultados esperados para el rumbo de la empresa. Es un factor fundamental en el liderazgo, administración y dirección de las empresas".

El quinto factor (promoción), como lo menciona la revista de recursos humano (2011) "La promoción es el traspaso de un empleado de un puesto de trabajo a otro al que le corresponde mayor salario, mayor autoridad y responsabilidad, un nivel más elevado en la organización, o varios de esos aspectos. Al promocionado le supone una mejora en su situación económica y social". En definitiva, mayor prestigio. La promoción se produce por dos razones conjuntas: reconocimiento a los resultados obtenidos en el pasado, y como esperanza en un prometedor futuro.

\section{MÉTODO}

La investigación que en este documento se planteó un enfoque cuantitativo, con un análisis descriptivo y recorte transversal, se apoyó de una encuesta, la cual se denominó: "Bienestar laboral", asimismo para la concentración de la base de datos se empleó una recolección en base a la técnica de conveniencia, pues es la estrategia más efectiva para reunir a todos los sujetos de estudio, tal y como lo indica Roberto Sampieri (2016) acerca de estudios con enfoque cuantitativo.

El diseño metodológico se definió en 3 fases: la primera fue la selección de docentes (FCA) de una Institución de educación Superior (IES) en la región sur del estado de Veracruz, la segunda fue la revisión teórica, selección y adecuación del cuestionario; la tercera se enfocó en la aplicación de la encuesta a docentes de IES en el sistema escolarizado de esta institución. Por tanto, se integraron la información correspondiente 
para su inferencia. En este sentido, se determinaron estrategias que esta institución de IES deberían de seguir para tener más motivados a los docentes e incidir en su práctica docente.

La variable central de esta investigación fue "Factores del Bienestar Laboral" en docentes de una IES en la región sur del estado de

\subsection{Planteamiento del problema}

Cada persona es distinta y posee cualidades tanto buenas como malas que se desarrollan a través de su vida, muchas de ellas son afectadas por diversos factores; pero sin duda lo que más le preocupa al personal docente de esta institución educativa Superior (IES), es contar con un nivel aceptable de bienestar en su vida laboral al interior de su centro de trabajo y en los resultados de su práctica docente. Es por ello que los indicadores referentes que harían suponer niveles bajos de bienestar laboral en los docentes en el periodo febrero- julio 2020, son los siguientes: Fortalecimiento de personal docente de Tiempo completo por programa educativo, Actualización de Planes y programa por parte del PTC.

El porcentaje más alto se encontró con un valor de $90 \%$ en los docentes de tiempo completo (PTC9 que se interesan en la actualización de los planes y programas de estudio de las diversas asignaturas que conforman los diversos programas educativos que se imparten en esta institución de educación superior. (Universidad Veracruzana, 2021)

Por otra parte, los valores más bajos se presentan con un valor de $25 \%$ y $50 \%$ durante los años 2019 y 2020, este rubro hace referencia a incrementar el número de PTC por programa educativo de acuerdo al indicador establecido por la ANUIES.

Derivado de lo anterior, se presenta la necesidad de identificar qué factores están presentes en el bienestar laboral de los docentes de la facultad de contaduría y
Veracruz, y surge analizar los factores de esta variable; ya que se supone que los docentes de educación superior deben contar con bienestar laboral al ejecutar los factores de esta variable de estudio, con esto se mejora las funciones docentes en la facultad donde se desempeñan los sujetos de estudio (FCA).

administración en la universidad veracruzana campus Coatzacoalcos, con el objetivo de obtener un diagnóstico que permita conocer la situación actual en la institución, y posteriormente poder dar recomendaciones $y / 0$ estrategias para mejorar el bienestar laboral en los docentes.

\subsubsection{Objetivo general}

Analizar los factores de bienestar laboral en los docentes de la facultad de contaduría y administración de la Universidad Veracruzana (UV), en el periodo febrerojulio 2020.

\subsubsection{Objetivos específicos}

- Identificar la existencia del factor logro en las y los docentes de FCA

- Mostrar la presencia de reconocimiento en las y los docentes la UV.

- Analizar el factor trabajo en sí en las y los docentes de esta IES Pública.

- Determinar si existe el factor de responsabilidad en las y los docentes de esta Facultad (FCA).

- Presentar el factor promoción en los docentes de FCA en la Universidad Veracruzana.

\subsubsection{Justificación}

El resultado de esta investigación facilita a los actores del proceso (dirección, docente, estudiante), clarificar el panorama en la 
problemática que se vive al interior la facultad de FCA; se pretende lograr una descripción más profunda sobre los factores de motivación de bienestar laboral en el personal docente de FCA de la Universidad Veracruzana.

El diagnóstico servirá para diseñar un programa estratégico, esto para incidir sobre los niveles de logro en los factores de bienestar laboral del personal docente de FCA, y en el caso de la autorización, la implementación del mismo; así como la evaluación de los resultados derivados del programa.

A la dirección de UV (FCA), le proporciona bases objetivas, para una efectiva toma de decisiones relacionadas con el diseño de estrategias que incidan en los factores de bienestar laboral en su personal docente; para ello deberá de tomar en cuenta las necesidades actuales que resulten de esta investigación.

Los principales beneficiados serán los alumnos de los diversos programas educativos que se imparten en FCA, a partir de las acciones que se implementen a largo plazo, podrán incidir también en los niveles de bienestar educativo, pues estas se van a focalizar en donde realmente se necesita mejorar en su proceso de educativo.

Los docentes, son pilares claves en el proceso de su bienestar laboral, además de que son los que llevan a ese camino del logro a través de sus propios esfuerzos, en las diferentes experiencias educativas que imparten en los diversos programas educativos de la FCA. Por ende, les permite tener un panorama de la problemática a la dirección de la universidad, además de la implementación de estrategias necesarias para mejorar en sus clases con sus respectivos grupos e indicadores de desempeño solicitados por la IES pública.

El valor teórico de la investigación radica en la comprobación de los factores de bienestar laboral en el contexto educativo superior, la utilidad metodológica es la propuesta de un instrumento de medición que valore los factores del bienestar laboral en el personal docente de la FCA.

\subsection{Delimitación del problema}

En este sentido, se analizan los factores de bienestar laboral en el personal docente de educación superior público, la cual se encuentra basada en la Teoría del Bienestar laboral. Se realiza en la Universidad Veracruzana en el periodo agosto-diciembre 2020. Por lo tanto, se ratifica que utilizó una secuencia estructurada para su análisis cuantitativo, con apoyo de la técnica de encuesta, la cual mide los factores de bienestar laboral en docentes de educación superior en el sistema escolarizado.

\subsection{Población y muestra}

Para la determinación de la población se contactó a los docentes de la facultad de contaduría y administración, del ciclo escolar febrero- julio 2020, se alcanzó una recopilación de 59 docentes activos, de los cuales 53 lograron contestar, considerando al total de respuestas como población y no el de una muestra.

\subsection{Instrumento de recopilación}

El cuestionario que se utilizó para esta investigación fue el titulado "Bienestar laboral", este fue estructurado para su aplicación individual a los docentes. El cuestionario se responde en aproximadamente de 4 a 7 minutos, cuenta con 25 ítems, y se responde con una escala de Likert para los cinco factores de bienestar laboral docente, donde 1 representa el valor más bajo y 3 el más alto. Además, se incluyen los ítems considerados en el cuestionario para la valoración de cada uno de los factores de dicho instrumento Los factores que se analizan en el instrumento se visualizan en la tabla 1. 
Tabla 1. Factores de Bienestar laboral, items, escala Likert.

Fuente: elaboración propia, 2020.

Como se indicó el instrumento evalúa los factores de bienestar laboral de los docentes de FCA de la UV en el sistema escolarizado, el cuestionario se basó en una prueba psicométrica de psicología organizacional de México. Este cuestionario se adecuó a las necesidades del contexto en docentes de esta IES.

\section{RESULTADOS}

Enseguida, se mencionan los principales resultados encontrados en el realización de esta investigación, la recolección de los datos se realizó a partir de la aplicación de la encuesta de "Bienestar laboral " aplicado a docentes de FCA del UV, el proceso de aplicación del cuestionario fue tardío, sin embargo para la fiabilidad se usó la técnica de alfa Cronbach con apoyo del programa estadístico SPSS V. 25, el cual obtuvo una valoración de 0,856 en general tal y como se aprecia en la tabla 2.

Tabla 2. Estadístico de confiabilidad del Bienestar laboral docente $U V$.

\begin{tabular}{|l|l|}
\hline \multicolumn{2}{|c|}{ Estadística de fiabilidad } \\
\hline $\begin{array}{l}\text { Factores de } \\
\text { Bienestar laboral }\end{array}$ & Alfa de Cronbach \\
\hline F1. Logro & .846 \\
\hline F2. Reconocimiento & .865 \\
\hline F3.Trabajo en sí & .844 \\
\hline F4.Responsabilidad & .856 \\
\hline F5. Promoción & .875 \\
\hline & $\mathbf{. 8 5 6}$ ( 25 ítems) \\
\hline
\end{tabular}

El valor general del alfa de Cronbach, según lo enunciado por Sierra Bravo (2001) es analizado como fuerte, sin embargo, este autor infiere que la interpretación de confiabilidad debe de ser empelado en cada

\begin{tabular}{|l|l|}
\hline \multicolumn{1}{|c|}{$\begin{array}{c}\text { Factores de } \\
\text { Bienestar Laboral }\end{array}$} & Ítems \\
\hline Logro & 5 \\
\hline Reconocimiento & 5 \\
\hline Trabajo en sí & 5 \\
\hline Responsabilidad & 5 \\
\hline Promoción & 5 \\
\hline
\end{tabular}

una de los factores e items que estructuran el cuestionario, por ello se obtuvo el estadístico de confiabilidad por factor y el de menor valor fue 0,844 (F3) y el de mayor 0,875 (F5), de cada una de las dimensiones el nivel es muy fuerte según el autor, además se hizo un análisis para cada ítems el cual se encontró que los 25 están dentro del mismo parámetro. Es necesario indicar que el cálculo del estadístico de fiabilidad da por hecho la confiabilidad de los datos recolectados, incluyendo la consistencia interna de dicho cuestionario de recolección.

Por otra parte, el análisis de la tabla 3 se establecen los porcentajes en los datos generales de los docentes de IES; los resultados determinan que; $45.29 \%$ son féminas, el 54.71 son docentes masculinos; el $69.81 \%$ tienen una relación civil familiar, sin embargo, en esa misma variable se observa que el $20.75 \%$ tienen un status de soltero este resultado es relevante, ya que determina que la mayoría de personas que ingresan a laborar en la educación superior son docentes jóvenes, asi como $45.29 \%$ de los encuestados se encuentra con una basificación dentro de la FCA, lo que facilita un área de oportunidad del $54.71 \%$ en esta meta laboral.

Tabla 3. Datos sociodemográficos de los docentes encuestados.

\begin{tabular}{|l|l|l|}
\hline & Frecuencia & Frecuencia \\
\hline
\end{tabular}




\begin{tabular}{|c|c|c|}
\hline & absoluta & relativa \\
\hline Masculino & 29 & $54.71 \%$ \\
\hline Femenino & 24 & $45.29 \%$ \\
\hline \multicolumn{3}{|c|}{ Estado civil } \\
\hline Casado (a) & 37 & $69.81 \%$ \\
\hline Soltero (a) & 11 & $20.75 \%$ \\
\hline Divorciado (a) & 4 & $7.54 \%$ \\
\hline Unión libre & 1 & $1.90 \%$ \\
\hline \multicolumn{2}{|c|}{ Tipo de contrato } \\
\hline Planta & 24 & $45.29 \%$ \\
\hline $\begin{array}{c}\text { Interino por obra } \\
\text { determinada }\end{array}$ & 29 & $54.71 \%$ \\
\hline
\end{tabular}

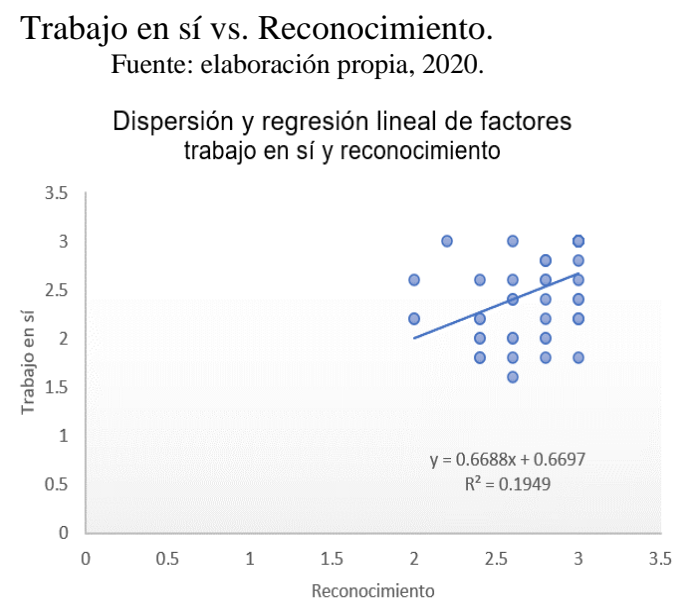

Fuente: elaboración propia, 2020.

Además, existe una alta matriculación de este último para este género docente, es decir que los varones son los que prefieren en mayor medida dedicarse a la docencia en el nivel licenciatura de FCA.

Lo anterior, confirma que al ingresar a laborar a una institución de educación superior en los programas educativos que ofrece FCA, el género no determina diferencia para su vida laboral al interior de la Universidad, pues a su ingreso y permanencia en la enseñanza de nivel superior, se podrán incorporar al sector educativo con las mismas ventajas y oportunidades, todo esto en función de los perfiles académicos de las diversas experiencias educativas que se ofrecen en esta IES.

En la figura 2, se puede apreciar el nivel promedio del factor Trabajo en sí vs. el nivel del factor reconocimiento de los docentes, se observa que la mayoría afirma una relación significativa entre ambos factores, gran número se encuentra por arriba de la media global, lo que indica que según la escala es: favorable, es decir, cuentan con el bienestar de sus propios esfuerzos en el trabajo y los incentivos proporcionados por la universidad son proporcionales al mismo, es decir lo que han aplicado en su práctica docente(FCA).

En la figura 3 , se mencionan los resultados de medias de los factores: Logro vs. su nivel de responsabilidad de los docentes de FCA en el desempeño de sus funciones, para su interpretación se usará la escala valorativa de este instrumento donde: (1) Nunca, (2) Algunas Veces, (3) Siempre.

Figura 3. Gráfica de dispersión y correlación Logro vs. Responsabilidad.

Figura 2. Gráfica de dispersión y correlación 


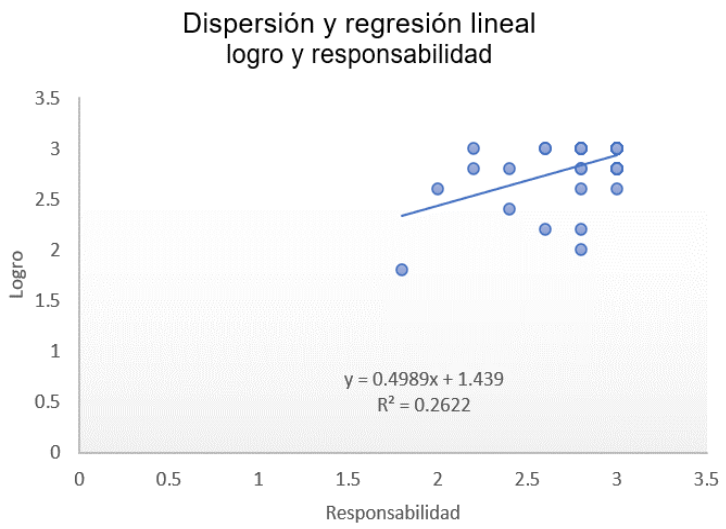

Fuente: elaboración propia, 2020.

Primero se hará un análisis de cada factor, con respecto a la F1 la media es 2.84 que según la escala se interpreta que; los docentes de FCA están conscientes de que es importante el logro académico al momento del ingreso y permanencia del nivel superior, esto en relación a cada uno de los componentes relacionados. Con respecto a la F2 la media fue 2.51 y se interpreta puntualmente igual, que los docentes asimilan actividades de reconocimiento que la universidad les ofrece por el cumplimiento de diversos indicadores propios de la IES.

La F3 que se refiere al nivel de trabajo en sí los docentes de FCA desde su percepción y autoevaluación, la media obtenida fue de 2.75 en una escala del 1 al 3, en donde 1 es el valor más bajo y 3 el más alto, la cual indica que los docentes tienen una valoración de trabajo en sí como buena en el desarrollo de su práctica docente.

Por otra parte, la F4 hace énfasis a la responsabilidad que los profesores de la IES pública durante su vida laboral al interior de la universidad, este factor se presenta con un valor de 2.96. Es decir, los docentes de FCA reconocen su responsabilidad como favorable en el cumplimiento de sus metas académicas.
En el caso de la F5 que señala el factor de promoción en los docentes de esta Universidad educativa infiere una media de 2.62. Lo que se interpreta que dichos profesores de FCA se encuentran satisfechos con el programa de promoción vertical y horizontal que la IES les ofrece por pertenecer al personal que tienen en contrato temporal o definitivo.

Ahora bien, en segundo análisis, es conveniente realizar un comparativo por factores relacionados del bienestar laboral de los docentes de FCA, en la figura 2 y 3 que se mostró antes, se puede apreciar que el reconocimiento y trabajo en sí, poseen casi el mismo valor al momento de su valoración, sin embargo, los resultados demuestran que la variación es mínima entre ambos factores de bienestar. Para un mejor entendimiento del comparativo por dimensión en la figura 4 se puede apreciar cómo se encuentra el grado de bienestar de los docentes de FCA, los promedios se calcularon a partir de las medias extraídas por género y se le aplicó una sumatoria de ambos valores de los datos y dividirlo entre dos. para obtener los resultados correspondientes, estos se interpretan en la misma escala valorativa de las medias para los cinco factores del bienestar.

Figura 4. Gráfica de las medias en los factores de bienestar laboral de los docentes de FCA.

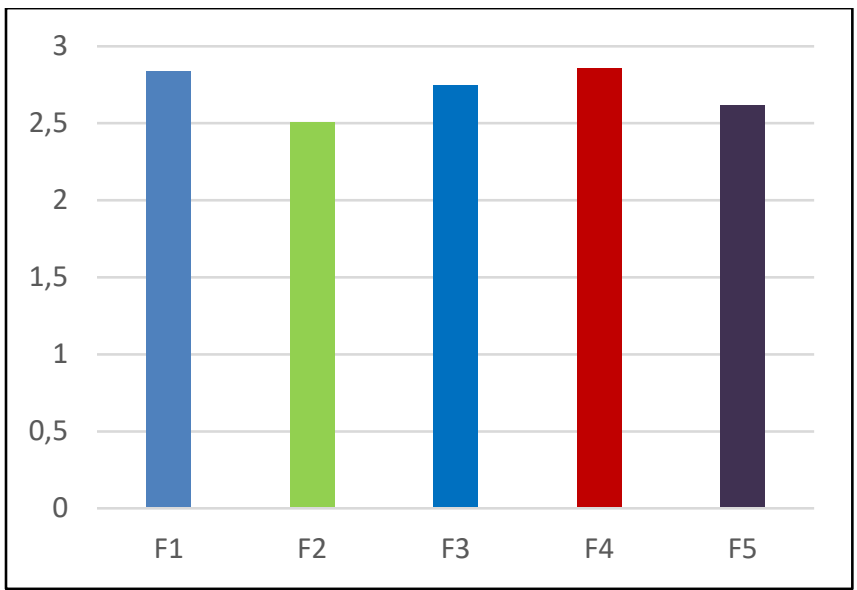

Fuente: elaboración propia, 2020. 
Los niveles obtenidos en cada factor demuestran que los docentes de FAC de la UV cuentan con bienestar favorable durante el ingreso y permanencia a este nivel educativo, además en la figura 4 se indica que el nivel general es de 2.72, lo cual es muy bueno ya que confirma una congruencia entre los valores individuales y general en los factores de su bienestar. Sin embargo, existen áreas de oportunidad ya que el nivel podría ser mejor, por ello es necesario que la institución de educación superior del sistema escolarizado implemente estrategias para mejorar.

En general, los docentes de FCA de la UV indican estar en satisfechos con el rendimiento de los factores de bienestar laboral, desde su percepción al ingresar y permanecer en este nivel educativo. Esto indica que, aunque algunos docentes en algunos momentos de su vida laboral al interior dela universidad, no encuentren la satisfacción de dichos factores, estos deberán en enfocarse para el cumplimiento de cada una de ellas de acuerdo a sus propios intereses y necesidades, concluyendo que el bienestar laboral es necesario para que todos los docentes lleven a cabo su proyecto de vida laboral con notas favorables en su propio desempeño.

\subsection{Análisis critico}

Los factores de bienestar laboral son necesarios que las ejecuten y racionalicen cualquier docente que desee ingresar $y$ permanecer en la FCA. En este estudio se recaba información general de los encuestados que vale la pena resaltar, por lo que respecta a la variable género, el $45.29 \%$ son mujeres y el resto son hombres. De ahí resulta que las y los profesores están igual de satisfechos al momento de percibir los factores presentes en su vida académica en la IES.

Los resultados se sometieron a una prueba de medias para identificar diferencias debidas al género, para los factores trabajo en sí vs. reconocimiento. En el caso de la ejecución del trabajo en sí, no existe diferencia en la percepción que tienen las y los estudiantes. Por otra parte, las profesoras se encuentran más reconocidas al ejercer sus funciones docentes al interior de FCA.

Además, al calcular el coeficiente de Pearson en el factor logro vs. responsabilidad de los docentes de FCA, salieron con una correlación positiva débil, es decir que, en estos factores no se consideran variables como la familia, salud, el resto la conforman otros factores externos como la situación económica etc, porque no formaron parte de este trabajo de investigación.

Los resultados antes descritos solo afirman que los docentes de FCA de la UV, racionalizan y ejecutan los factores de bienestar laboral, sin embargo, los resultados indican que existen áreas de oportunidad, para poder lograr ello es necesario que cada docente focalice su atención en mejorar su propio bienestar para lograr una ventaja competitiva durante su ingreso y permanencia en su vida laboral al interior de la IES.

\subsection{Recomendaciones}

Los resultados obtenidos son favorables para los factores tratadas por lo que es factible identificar un nivel bueno de bienestar laboral en los docentes de FCA de la UV. Sin embargo, existen áreas de oportunidad y para ello se plantean las siguientes recomendaciones:

- Establecer reuniones periódicas informativas con los docentes para informar resultados periódicos, y posterior a ello en las diversas coordinaciones de colegiado se diseñen las estrategias de solución específicas.

- Actualizar el programa de incentivos económicos y/o morales del personal 
docente de la FCA.

- Después de revisar las evaluaciones docentes realizados por los estudiantes cada ciclo escolar, se propone a la institución reforzar con cursos de actualización docente para la mejora del mismo periódicamente.

- Se propone definir programas de corto y mediano plazo para que los docentes identifiquen los puntajes que deben tener en productividad en función de los años de antigüedad que cuente en la universidad. Esto debe depender del tipo de contrato que los docentes ostenten al interior de FCA.

- Mejorar su programa de retribución económica por metas y objetivos alcanzados.

En general, algunas recomendaciones van dirigidas a los docentes de FCA de la UV, sin embargo, esta investigación busco analizar si estos ejecutan o no los factores de bienestar laboral al ingreso y permanencia de este nivel educativo, por ello uno de las recomendaciones para los investigadores es realizar estudios longitudinales, que permitan conocer si estos van mejorando o no.

\section{CONCLUSIONES}

El estudio realizado tuvo como propósito identificar los factores de bienestar laboral en los docentes (FCA), así como la determinación de estrategias para la mejora de la productividad de los docentes de esta universidad $(U V)$.

Cabe destacar que se cumplió el objetivo general, acerca de determinar el grado de los factores de bienestar laboral en docentes de FCA de la UV, se obtuvo que el nivel que ejercen es favorable. Aunado a ello, se cumplieron cada uno de los objetivos específicos, se alcanzaron y se pudo realizar un análisis por cada una de las características del docente con la percepción de su propio bienestar.

Los análisis por cada una de las características revelaron que el bienestar laboral de los docentes no se ve afectado por su tipo de contratación, situación sentimental. Es decir, ninguna de estas características individuales genera una limitación para continuar con su vida laboral (práctica docente). En el caso del género, las docentes brindan un mayor valor al factor de reconocimiento.

Sin embargo, se pudo afirmar que la mayoría de los docentes de FCA presentan algún tipo de soporte contractual, eso denota que el plantel tiene docentes comprometidos y responsables en su práctica docente, al mismo tiempo generan las metas académicas solicitados por la Universidad. Esos resultados llevaron a plantear recomendaciones pertinentes que pueden servirles a los docentes para autoevaluarse con un mejor nivel de bienestar.

En conclusión, la importancia de investigar este tipo de temas, es que permite a las organizaciones obtener resultados en el grado de bienestar laboral de las personas que la integran, con bases objetivas. A partir de ello, se originan una serie de estrategias precisas para mejorar en el nivel de bienestar, es decir, esto repercutirá en la productividad académica de la institución que realice dicho estudio. 
6.

\section{REFERENCIAS}

\section{Libro en línea}

B, E., Bauer, T., \& Mansfield, L. (2012). Whistle white you word: a reviewof the life satisfaction literature.

Libro en línea

Brief, A. P. (1998). Attitudes in and around organizations.

Libro en línea

Diccionario real de la lengua española. (2006). México.

Noticia o publicación en página de internet

Hernandez, E., \& Moreno, B. (2013). Salud Laboral. Madrid.

Artículo científico

humanos, R. d. (2019). Percepción laboral y su importancia dentro de las organizaciones. Redacción de recursos humano.

Artículo científico

Infocop. (2017). El papel del bienestar en el ámbito laboral: fundamentos y aportaciones desde la Psicología. revista de psicologia, 1-9.

\section{Artículo científico}

M B, J., S, M., C, L., \& Genis, C. (2010). Cuestionario de Bienestar Laboral General: Estructura y Propiedades Psicométricas. Revista de Psicología Del Trabajo y de Las Organizaciones.

\section{Libro impreso}

Maslow, A. (1991). Motivación y personalidad. España: McGrawHill Interamericana Editores, S. A. de C. V. $5^{\text {a }}$. Edición.

Noticia o publicación en página de internet

Parker, G., McAdams, J., \& Zielinski, D. (2013). Planes de reconocimiento de diversas compañias. Madrid: Diaz santos.

Noticia o publicación en página de internet

Preciado, A. C. (2006). Modelo de evaluación por competencias. Mexco D.F: Publicaciones cruz O S.A.

\section{Artículo científico}

Pujol, L. J. (2016). Satisfacción Laboral en docentes universitarios: medición y estudio de variables influyentes. Revista de docencia universitaria, 262.

\section{Libro en línea}

Rodgers, G., Lee, E., Swepston, L., \& Van, J. (2009). La Organización Internacional del Trabajo y la lucha por la justicia social. La Organización Internacional del Trabajo, 99.

\section{Libro impreso}


Sampieri, R. (2016). Metodología de la investigación. México: Mcgraw Hill.

\section{Libro en línea}

Serrat, M. A. (2017). Liderando el bienestar laboral. Barcelona: JM Boschet editor.

Informes y reportes gubernamentales, institucionales o empresariales

Universidad Veracruzana. (2021). PLADEA 2017-2021. Obtenido de https://www.uv.mx/coatza/admon/nosotros/pladea/

\section{Artículo científico}

Zapata, P., Peralta, M. J., \& Fernandez, D. P. (2014). Influencia de variables organizacionales en la calidad de vida laboral de funcionarios del sector público. Chile. 
Vol. 7 Núm. 2 